\title{
Slow to heal or slow to diagnose cancer?
}

\author{
Charlotte A. Richards, ${ }^{\star 1}$ Alice Cameron, ${ }^{2}$ John Collin, ${ }^{1}$ Ceri W. Hughes ${ }^{1}$ and Barry G. Main ${ }^{1,3}$
}

\section{Key points}

Describes two cases of delayed referral of oral squamous cell carcinoma and their clinical progression
Outlines key learning points to help dental practitioners detect lesions requiring urgent referral.
Provides the reader with an overview of oral cancer including risk factors, signs and symptoms.
Emphasises why early referral is essential for improved prognosis.

\begin{abstract}
There are around 8,500 new cases of oral squamous cell carcinoma (SCC) diagnosed in the UK each year and the incidence appears to be increasing. Although surgical and non-surgical treatment options have improved significantly in the last few decades, five-year survival has not, with an average five-year survival of $56 \%$ in the UK. Providing patients have access, there are frequent opportunities for oral cancer screening by their general dental practitioner. We present two cases of SCC managed in our department, both of whom were referred following a protracted period of review in general dental practice, where the initial 'benign diagnoses' were not re-evaluated during this time. This delay can considerably impact on patient outcomes, including survival. We outline each patient's clinical course, and key learning points to help dental practitioners detect lesions that require prompt referral to the oral and maxillofacial service for urgent investigation.
\end{abstract}

\section{Introduction}

In one month in 2020, the oral and maxillofacial team in Bristol saw two patients via the 'two-week wait' (2WW) referral pathway who had received treatment for painful or swollen intraoral lesions by their general dental practitioners (GDPs) over a number of months before referral. These patients were subsequently diagnosed with oral squamous cell carcinoma (SCC). We present these two cases to illustrate the importance of considering referral for urgent assessment where a seemingly benign lesion progresses or fails to heal despite efforts at management.

'Oral and Maxillofacial Surgery, University Hospitals Bristol and Weston NHS Foundation Trust, Bristol, UK; ${ }^{2}$ Oral and Maxillofacial Surgery, Royal United Hospitals Bath Foundation Trust, Bath, UK; ${ }^{3}$ Centre for Surgical Research, Department of Population Health Sciences, Bristol Medical School, University of Bristol, UK.

*Correspondence to: Charlotte Richards

Email address: charlotte.richards@uhbw.nhs.uk

Refereed Paper.

Accepted 8 September 2020

https://doi.org/10.1038/s41415-021-2837-y

\section{Case one}

A 70-year-old female recent ex-smoker was urgently referred regarding a long-standing non-healing ulcer on the upper right buccal mucosa. Some eight months previously, she had two teeth extracted from the upper right quadrant and required additions to her denture. Three weeks after the addition, she developed a sore area, which was attributed to trauma from the new denture. The denture was eased and she was advised to use saltwater mouthwashes. She re-attended the same dentist five times with the same symptoms, with no resolution of the discomfort. She underwent further denture eases and eventually removal of the teeth from the denture, but the lesion persisted. On review by a different dentist, an urgent referral was completed.

On presentation, she had a $2 \mathrm{~cm}$ ulcerated lesion on the right buccal mucosa (Fig. 1). Histopathological examination confirmed a well-differentiated SCC with superimposed candidiasis. After discussion within the head and neck oncology multidisciplinary team, she elected to undergo a wide local excision, right selective neck dissection and reconstruction with a radial forearm free flap.

Histological analysis confirmed welldifferentiated SCC excised with clear margins and final tumour/node/metastasis (TNM) staging pT1 N0 M0. ${ }^{1}$ There were no postoperative complications; she did not require radiotherapy and is beginning five years of close clinical surveillance.

\section{Case two}

A 62-year-old male non-smoker, with moderate alcohol intake, was referred by his dentist following a ten-month history of gum problems in the upper left quadrant. He presented to his GDP with swelling and soreness in the upper left premolar area. He was prescribed chlorhexidine mouthwash, which initially reduced the swelling, but a month later he returned with the same symptoms. He was prescribed further mouthwash, but things did not improve and he was subsequently prescribed a course of metronidazole for a 'gum infection'.

Four months later, he re-attended complaining of loose teeth. A periapical 
radiograph of the upper left premolars showed $100 \%$ bone loss (Fig. 2) and the two teeth were extracted. Following the extractions, he re-presented to his dentist several times over a six-month period due to a 'non-healing socket'. He had also reported unintentional weight loss. An urgent referral to the maxillofacial team was made at this point.

On presentation, he had a large ulcerative, mixed red and white destructive lesion affecting the left maxilla that extended to the midline (Figures 3 and 4). There were no palpable lymph nodes. Histopathological analysis confirmed invasive moderately to poorly differentiated SCC, which was staged clinically as T4 N0 M0.

He subsequently underwent a left hemimaxillectomy, left neck dissection and reconstruction with a radial forearm free flap. Histopathological examination of the excised tumour and neck dissection confirmed a T4 tumour with close margins and metastatic disease in the cervical lymph nodes, with extra nodal extension (T4 N3b Mx). Due to the size of the primary tumour and extra nodal extension, he was offered radical post-operative radiotherapy. Unfortunately, the radiotherapy planning scans revealed pulmonary metastasis, meaning that radical radiotherapy was no longer viable and palliative chemotherapy was not available due to the concurrent COVID-19 pandemic. The patient died of his disease four months after his referral.

\section{Discussion}

\section{Oral cancer epidemiology}

In 2019/2020, 8,337 people were diagnosed with oral cancer in the United Kingdom. ${ }^{2}$ The average five-year survival rate is $56 \%{ }^{3}$ Smoking and alcohol are well-established risk factors, with combined alcohol intake and tobacco smoking having been shown to increase the risk of oral cancer by five times, due to their synergistic effect. ${ }^{4}$ Despite there being a significant decrease in smoking in the UK since the 1980 s, we have seen an increase in incidence of oral cancer, and with that, a shift in the aetiology of oral cancers, with an increasing incidence of oral cancers associated in females who have never smoked. Human papillomavirus is also an important aetiological factor in oropharyngeal (tonsillar and base of tongue) SCC. ${ }^{5}$ Age is a risk factor, with most cases being diagnosed between the age of 60 and $69 .^{6}$

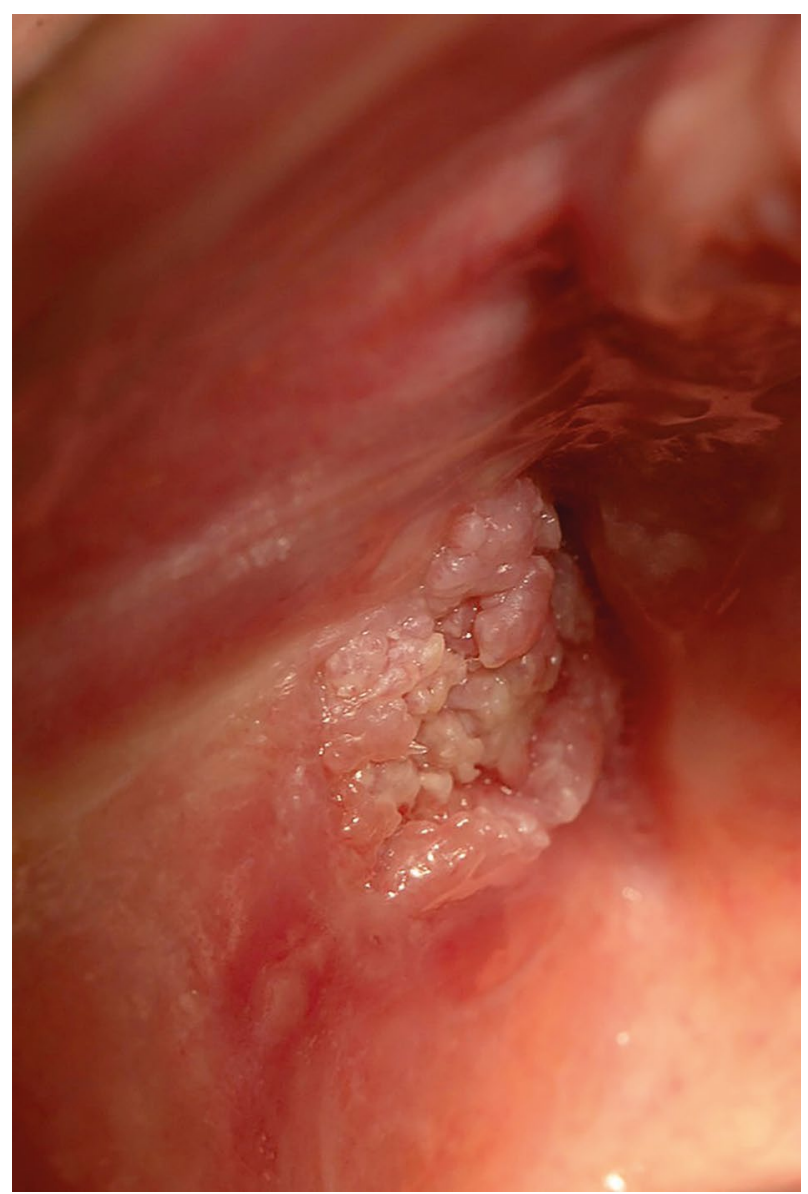

Fig. 1 Squamous cell carcinoma right buccal mucosa. Speckled appearance and rolled margins. Note edentulous upper ridge where denture teeth were added

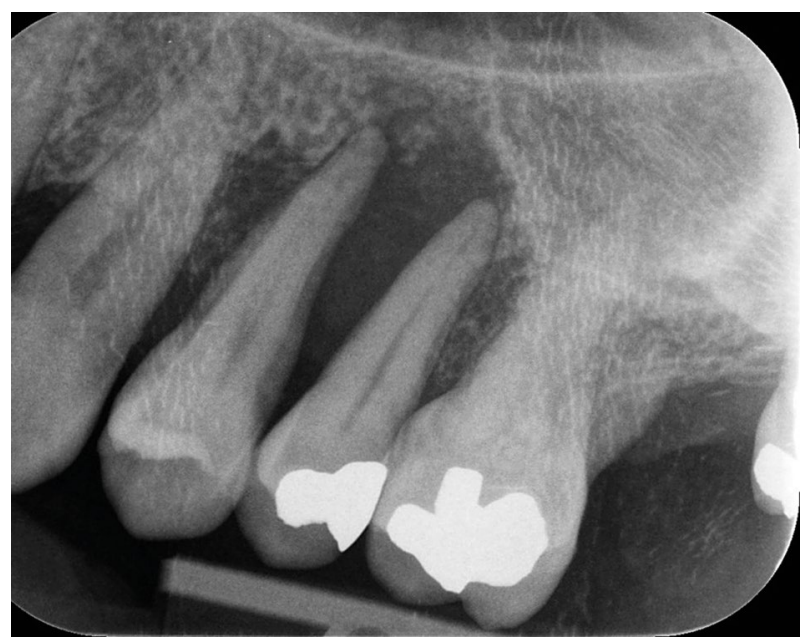

Fig. 2 Periapical radiograph of 23, 24, 25 and 26, irregular, severe bone loss associated with 24 and 25 , with bone loss extending to mesial of 23

The five-year survival rates for oral cancer vary depending on the stage at which it is detected. The best outcome is for a stage I small $(<2 \mathrm{~cm})$ tumour with no metastatic spread, with an average five-year survival of $80 \%$. This survival rate halves to only $40 \%$ for stage III tumours that are larger than $4 \mathrm{~cm}$. Tumours that have metastasised (stage IV) have an even worse prognosis with an average $10 \%$ fiveyear survival rate. ${ }^{3,7}$ Given the dramatically reduced prognosis for larger tumours, GDPs play a vital role in detecting cancer at an early 


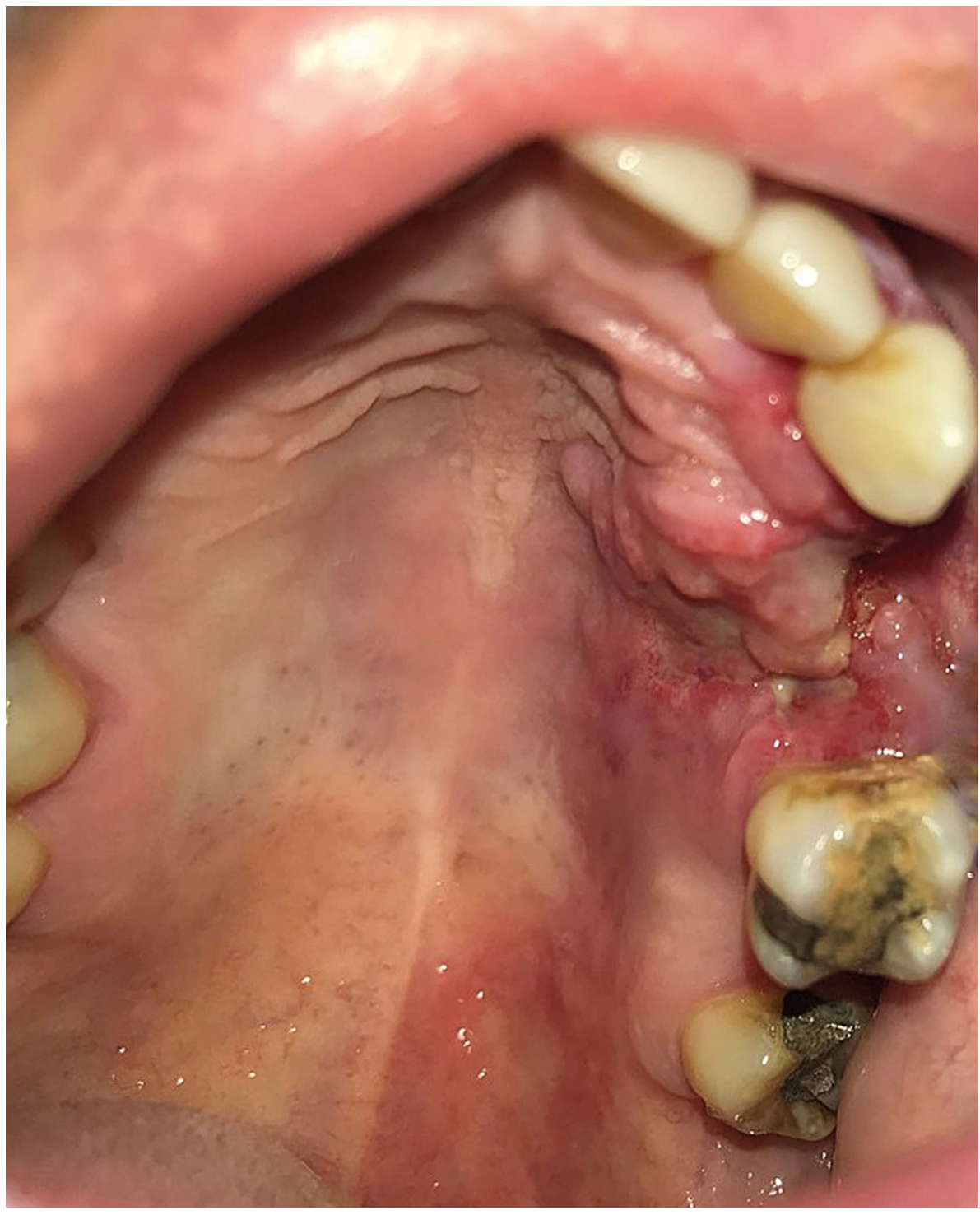

Fig. 3 Squamous cell carcinoma left maxilla. Ulcerative, mixed red and white destructive lesion, with erythema extending across the entire left hard palate

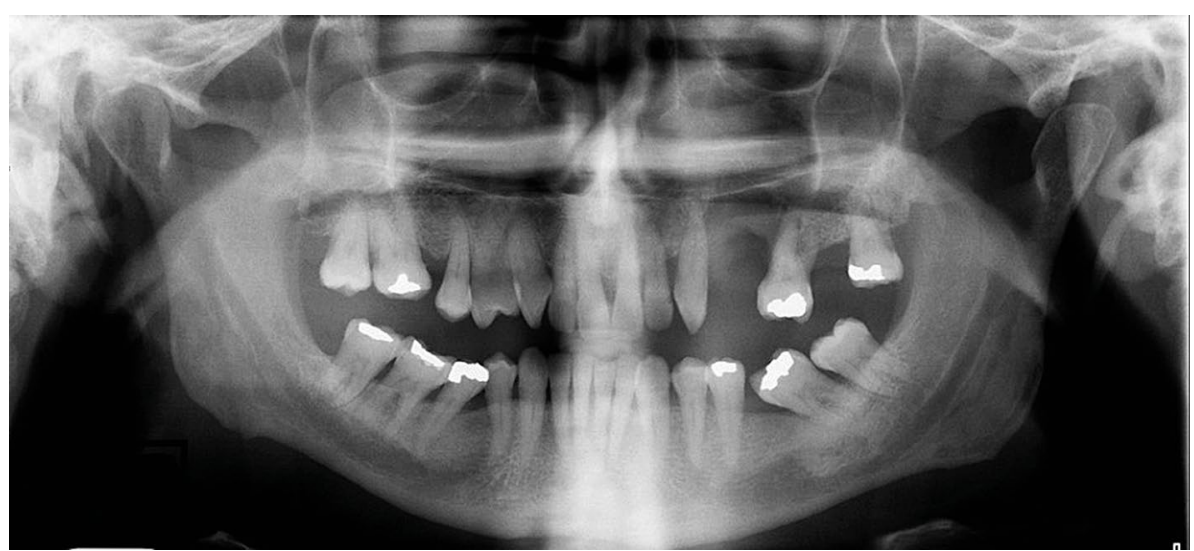

Fig. 4 An OPG radiograph showed a radiolucency on the ridge between the 23 and 26, with loss of antral base and generalised mild-moderate horizontal bone loss

stage. As well as improved overall survival, tumours detected at an earlier stage have reduced surgical morbidity, reduced need for a reconstructive flap necessitating intensive care admission and reduced likelihood of needing subsequent radiotherapy. Unfortunately, 35\% of patients diagnosed with oral cancer present at a late stage. ${ }^{2}$

\section{Signs and symptoms of oral cancer}

Ninety percent of oral cancers are SCCs, ${ }^{8}$ characterised by abnormal accelerated growth in the squamous cells of the oral epithelium. As this is the surface of the oral mucosa, any change should be visible. The classical appearance is a solitary non-healing ulcer, which is firm (indurated) and has rolled margins. Typically, this type of ulcer most commonly occurs on the tongue but may occur on any of the soft tissues. ${ }^{8}$ However, there is a broad spectrum of signs and symptoms (Table 1 ).

Case one represented a buccal mucosal SCC. The lesion was a long-standing mixed red and white patch which was non-responsive to local measures. Case two represented a maxillary SCC presenting as a speckled ulcerative lump with a mixed red and white appearance. Loose teeth are a sign of a late-stage tumour with bone invasion. Although the periapical radiograph, if viewed in isolation, could be representative of severe periodontal disease, when taken into context with the rest of the mouth and rapidly progressive tooth mobility, it is highly likely to represent a cancer. Anecdotally, the most commonly missed SCCs seen in the $2 \mathrm{WW}$ clinic are those where patients give a history of teeth suddenly becoming loose, the teeth are extracted and the patients then re-present over several months with non-healing. The oral cavity normally heals extremely effectively, so non-healing of an extraction site is likely to indicate underlying pathology such as infection or malignancy.

The primary care interval is the time taken from the patient initially presenting to a primary care clinician with symptoms relevant to the cancer diagnosis to the date of referral for suspected cancer. ${ }^{9}$ Both of these cases represent prolonged primary care intervals. The most challenging area of diagnosis in these cases is the thought process required to re-evaluate the diagnosis when the initial 'benign diagnosis' has been made, but the condition does not improve with the normal treatment for the 'benign diagnosis.' In comparison to general medical practitioners, it has been suggested there may be a slightly delayed primary care interval by dentists, due to them being more likely to undertake a dental procedure to address a 'benign' lesion and therefore delay the referral. ${ }^{10}$ In case one, 
the additions to the patient's denture and the resulting 'trauma' in the buccal mucosa adjacent to the addition is initially the most likely cause of the soft tissue trauma. It is important to book a clinical review following relief of the causative factor, and if the area has not improved within 2-3 weeks, reconsider the possible diagnosis and refer.

Sudden onset of tooth mobility should be treated with suspicion. This is easier to recognise in a patient who has regular checkups with the same dentist, via comparison of tooth mobility, periodontal pocketing or radiographs. The most common cause of tooth mobility is periodontal disease; however, this a slow, chronic process. Using case two as an example, the bone loss seen on the periapical radiograph is severe and irregular, in comparison to the generalised mild-tomoderate periodontal bone loss visualised on the orthopantomogram (OPG). It is important to consider whether the tooth mobility matches the picture of the whole mouth with generalised chronic periodontal disease or whether this is isolated to one to two teeth in one quadrant. There will also likely be associated soft tissue changes such as ulceration or erythema, which may extend into areas such as the hard palate or floor of mouth, which is unusual in chronic periodontal inflammation. A further example case from an indemnity provider found a breach in a dentist's care when a patient who was ultimately diagnosed with SCC of her mandible had undergone 18 weeks of review of a non-healing socket following extraction of a mobile lower molar tooth before a routine referral for assessment of presumed chronic periodontal disease being made by the dentist. A key point from that case is to consider how chronic periodontal disease might mask other acute presentations. ${ }^{11}$

The two cases presented demonstrate that the same type of cancer can have inherently different clinical behaviour (Table 2). This can be assessed under the microscope. A poorly differentiated tumour is one where the advancing front of the tumour is noncohesive, with infiltrating small islands, many abnormal mitotic cells and pleomorphism (that is, the cells bear no resemblance to normal squamous epithelium). Other poor histological prognostic factors are depth of the tumour, with a deeper tumour more likely to have regional lymph node involvement, perineural invasion and invasion of vessels. ${ }^{12}$ Despite both patients having a protracted clinical history before referral, they have

\section{Table 1 Signs and symptoms of oral cancer}

\section{Visual examination}

Ulcer ( $>3$ weeks, raised rolled edges)

Red lesion

Irregular white lesion

Mixed red and white lesion

Lump

Extraction socket not healing

\section{Physical examination}

Tooth mobility

Induration of lesion ('thickness' to tumour which can be felt between fingers)

Fixation of lesion to underlying tissue

Enlarged hard regional lymph node(s)

\section{History taking/often late symptoms}

Weight loss

Dysphagia

Dysarthria

Pain (late symptom, absence of pain does not exclude malignancy)

Paraesthesia (eg to lower lip when tumour has eroded inferior dental nerve)

Table 2 The presence of high-risk histological features in the tumours of the two cases

\begin{tabular}{l|l|l} 
Histological features & Case one - well differentiated & Case two-poorly differentiated \\
\hline Pattern of invasion & Cohesive & Non-cohesive \\
\hline Tumour depth & $4.5 \mathrm{~mm}$ & $10 \mathrm{~mm}+$ \\
\hline Perineural invasion & No & Yes \\
\hline Invasion of vessels & No & Yes \\
\hline
\end{tabular}

starkly different outcomes. In case one, the patient had a slow-growing tumour, which even at time of excision was less than 2 $\mathrm{cm}$. Given the small size, it is possible this may have initially presented to the GDP as an area of severe dysplasia or carcinoma in situ, which over the period of review transformed into SCC. In case two, the cancer demonstrated aggressive characteristics, with regional lymph node involvement at the time of surgery even though cross-sectional imaging had been negative only a few weeks prior. The earlier the patient is referred in the stage of tumour development, the higher the chance of cure.

\section{WW referral pathway}

In 2000, The NHS cancer plan introduced the $2 \mathrm{WW}$ rule to increase the speed that patients with suspected cancer are seen by a specialist. ${ }^{13}$ The National Institute for Health and Care Excellence (NICE) guidelines for oral cancer referrals prescribe which suspicious signs and/or symptoms require urgent referral. The referral pro forma which referrers use is based upon these. ${ }^{14}$ The South West Clinical Network referral form has the following boxes to be selected by referring clinicians as appropriate where there is a suspicion of oral cancer:

1. Unexplained ulceration of the oral cavity or mass persisting for more than three weeks

2. Unexplained red or white patch (including suspected lichen planus) of the oral cavity, particularly if painful, bleeding or swollen

3. Oral cavity and lip lesions or persistent symptoms of the oral cavity followed up for six weeks where a definitive diagnosis of a benign lesion cannot be made

4. Non-healing extraction sockets ( $>4$ weeks' duration) or suspicious loosening of teeth, where malignancy is suspected (particularly if associated with numbness of the lower lip). ${ }^{15}$

The current 2015 NICE guideline does not state point 4 in the referral criteria, despite this being the diagnostic criteria for case two. Interestingly, the previous 2005 guideline which has been superseded did include point 4 : 'unexplained tooth mobility not associated with periodontal disease.' In addition, the 'Scottish referral guidelines for suspected cancer' do not contain criteria regarding non-healing sockets or unexplained tooth mobility. ${ }^{16}$ 


\section{Conclusion}

The two cases presented highlight the need for vigilance and prompt referral of lesions that are suspicious for oral cancer. There are potentially serious consequences for patients if there are significant delays between the initial presentation of the lesion and referral for specialist opinion.

The key learning points for dental practitioners from our paper are:

- The most common site for SCCs is the tongue, ${ }^{3}$ but they can occur on any mucosal area including the maxilla

- Delay in referral can considerably decrease patient survival, particularly when referral is made at a late tumour stage

- The best possible scenario is referral when the tumour diameter is less than $2 \mathrm{~cm}$

- Thorough and systematic clinical examination of the soft tissues and examination of regional lymph nodes at each appointment helps early detection

- Once the initial management for a presumed benign local cause is unsuccessful and mucosal areas show no resolution, there should be an increased level of suspicion and reconsideration of possible diagnoses

- GDPs should refer cases of concern to their local oral and maxillofacial team early to help avoid the additional morbidities associated with the management of latepresenting tumours.

\section{Acknowledgements}

With thanks to both patients for giving permission for images to be published.

\section{Competing interests}

The authors declare no competing interests.

\section{References}

1. American Joint Committee On Cancer. Cancer Staging manual (8th ed). 2018. Available at https:// cancerstaging.org/references-tools/deskreferences/ Documents/AJCC\%20Cancer\%20Staging\%20 Form\%20Supplement.pdf (accessed May 2020)

2. Oral Health Foundation. State of Mouth Cancer UK Report 2019/2020. 2020. Available at https:// www.dentalhealth.org/Handlers/Download. ashx?IDMF=630bdca4-0634-44cd-b8dd8dab7b6f4d0a (accessed May 2020).

3. Cancer Research UK. Health professional. Available at https://www.cancerresearchuk.org/healthprofessional (accessed May 2020).

4. Mello F W, Melo G, Pasetto J J, Silva C A B, Warnakulasuriya S, Rivero E R C. The synergistic effect of tobacco and alcohol consumption on oral squamous cell carcinoma: a systematic review and meta-analysis. Clin Oral Investig 2019; 23: 2849-2859.

5. Thomas S, Penfold C, Waylen A, Ness A. The changing aetiology of head and neck squamous cell cancer: A tale of three cancers? Clin Otolaryngol 2018; DOI: 10.1111/coa.13144.

6. National Institute of Dental and Craniofacial Research Website. Oral Cancer Incidence (New Cases) by Age, Race, and Gender. 2018. Available at https:// www.nidcr.nih.gov/research/data-statistics/oralcancer/incidence (accessed May 2020).

7. Lewis M A O. Mouth cancer: presentation, detection and referral in primary dental care. Br Dent J 2018; 225: 833-840.

8. Scully C. Oral and Maxillofacial Medicine: The basis of diagnosis and treatment. 3rd ed. London: Churchill Livingstone; 2013.

9. Lyratzopoulos G, Saunders C, Abel G et al. The relative length of the patient and the primary care interval in patients with 28 common and rarer cancers. Br J Cancer 2015112 Suppl 1(Suppl 1): S35-S40.

10. Grafton-Clarke C, Chen K W, Wilcock J. Diagnosis and referral delays in primary care for oral squamous cell carcinomas: a systematic review. Br J Gen Pract 2019; 69: 112-126.

11. MDDUS. Slow-healing Socket. 2018. Available at https://www.mddus.com/resources/resource-library/ case-of-the-month/2018/june/slow-healing-socket (accessed May 2020).

12. Speight $P$, Farthing $P$. The pathology of oral cancer. $B r$ Dent J 2018; 225: 841-847.

13. Department of Health. The NHS Cancer plan: a plan for investment, a plan for reform. 2000. Available at https://www.thh.nhs.uk/documents/_Departments/ Cancer/NHSCancerPlan.pdf (accessed May 2020).

14. National Institute for Health and Care Excellence. Head and Neck Cancers Referral and Recognition. 2016. Available online at https://cks.nice.org. uk/head-and-neck-cancers-recognition-andreferral\#!scenario (accessed May 2020).

15. NHS England and NHS Improvement South West. Suspected Head and Neck Cancer Referral form 2 week wait. 2020. Available online at https://www. england.nhs.uk/south/info-professional/dental/dcis/ forms / (accessed July 2020).

16. Scottish Referral Guidelines for Suspected Cancer. Homepage. 2019. Available at http://www. cancerreferral.scot.nhs.uk/Home (accessed July 2020).

\section{Correction to: Book review: Orthodontics: diagnosis and management of malocclusion and dentofacial deformities, 3rd Ed}

The original article can be found online at https://doi.org/10.1038/s41415-020-2571-x

Author's correction note:

Book review article Br Dent J 2021; 230: 10.

When this article was initially published the second author's name was not included. The author list should have read:

'Sagar Dahiya \& Rajat Mitra'

The journal apologises for any inconvenience caused. 